\title{
Perception of the horizontal and vertical in tangible displays: minimal gender differences
}

\author{
Morton A Heller $\Phi$ \\ Winston-Salem State University, Winston-Salem, NC 27110, USA \\ Jeffrey A Calcaterra \\ Wright State University, Dayton, OH 45435, USA \\ Shavonda L Green\# \\ Winston-Salem State University, Winston-Salem, NC 27110, USA \\ Stevette L Barnette \\ Radford University, Radford, VA 24142, USA \\ Received 25 June 1997, in revised form 29 June 1998
}

\begin{abstract}
A study is reported of gender differences in a haptic version of three Piagetian tests that assess understanding of Euclidian space. A raised-line drawing kit was used both for subject responses and for production of stimuli. To test understanding of the horizontal, subjects felt pictures of a jar at four tilts and were asked to draw the water line. Two methods were used to examine understanding of the vertical. First, subjects drew a hanging electrical cord and light bulb, attached to the ceiling of a bus, parked on hills of four different angles. Subsequently, subjects drew telephone poles (represented by a single line) on hills of four different angles. In the jar task, males and females showed comparable performance, both groups showing large errors. Judgments of the vertical were very similar for males and females in the bus task, but errors diminished considerably for both genders when subjects drew telephone poles on hills. It is suggested that better judgments of the vertical in the pole task probably derive from the use of body-centered spatial reference information.
\end{abstract}

\section{Introduction}

The water-level and plumb-line tasks were proposed by Piaget and Inhelder (1956) to demonstrate the development of spatial reasoning about the horizontal and vertical in children. In these tasks, subjects draw a line to indicate the water level in a tilted jar or draw a hanging plumb line. The original version of the task required children to anticipate the water level on being given a tilted jar. Subsequently, Piaget asked children to draw the water level while looking at a real tilted jar with water in it. Piaget was interested in whether children would discover the principle that the water stays horizontal during an experimental session. In order to complete these tasks correctly, subjects must understand that the direction of the water level and the direction of the hanging plumb line do not vary as their containers are tilted. An understanding of the invariance of the horizontal and vertical is important for locating objects in Euclidian space, and processing pattern information.

Later studies have found that a surprisingly large proportion of college students fail at the tasks and, further, that males often show higher performance than females (Harris et al 1977; Robert and Harel 1996; Robert and Ohlmann 1994; Voyer et al 1995). Robert and her colleagues (eg Berthiaume et al 1993; Robert et al 1994) have claimed that the gender difference in the water-level task disappeared when subjects were given a haptic version of the task. In this task, subjects moved a magnetic bar to show the water level in a tangible jar, represented by a rectangular hole in a metal plate. Robert et al attributed the comparable performance of males and females to the lack of salience of

- Current address to which correspondence and requests for reprints should be sent: Department of Psychology, Eastern Illinois University, 119 Physical Sciences Building, 600 Lincoln Avenue, Charleston, IL 61920-3099, USA; e-mail: cfmah1@ciu.edu. 
irrelevant cues (ie the sides of the jar) when the task was done haptically. According to them, when the task is done visually, the oblique lines are more difficult to ignore.

Two methodological factors in these experiments served to make the reduced haptic salience argument less convincing. First, visually, subjects use a pencil to add a line to a drawing, using a familiar form of depiction. Haptically, subjects were required to perform a very different task - to imagine that the rectangular hole represented a jar, the solid plate represented empty space, and a magnetic bar represented the water line. Note that task requirements, involving manipulating the bar, would tend to draw subjects' attention away from the shape of the jar. Under these circumstances, subjects could not readily feel the hole representing the jar while manipulating the bar with both hands, and this may have helped them ignore the distracting framework representing the jar. Second, subjects were tested in room lighting, where touch has been shown to be less accurate for some spatial tasks involving imagery (Heller 1993). Conceivably, access to visual information about the vertical and horizontal in the room could have aided subjects and improved performance.

In the present study, subjects completed a haptic drawing version of the water-level task. Subsequently, subjects completed a haptic version of the plumb-line task. Finally, subjects completed an additional task for assessing the vertical-the telephone-pole task in which they were asked to make raised-line drawings of telephone poles on hills varying in angle. Males were expected to show superior performance in each of the tasks, if gender differences previously found in vision generalize to touch.

\section{General method}

\subsection{Subjects}

Subjects were twenty-four (twelve male, twelve female) undergraduate volunteers recruited on the Winston-Salem State University campus. Subjects were blindfolded throughout the experiments, and participated in each of the drawing tasks reported here.

\subsection{Stimuli and apparatus}

Durable raised-line drawings were produced with a Swedish raised-line drawing kit. The Swedish drawing kit produces a tangible and visible line when a ball-point pen is drawn over the surface (see Heller et al 1996a, 1996b; Heller and Schiff 1991).

2.2.1 Jar task. The jar was depicted as a rectangle, $3.5 \mathrm{~cm}$ wide by $7 \mathrm{~cm}$ high (the height included a $0.5 \mathrm{~cm}$ cap). The standard jar was drawn in its normal upright position, at $0^{\circ}$ (see figure 1). Jars were drawn at four different tilts: $-60^{\circ},-30^{\circ}, 30^{\circ}$, and $60^{\circ}$. Jars at negative angles leaned to the left of the vertical; ones at positive angles leaned to the right. The upright standard, at $0^{\circ}$, showed the jar half-full of water (with a horizontal line connecting the midpoints of the vertical segments). A 9-cm-long horizontal line was drawn at the bottom of each jar to show the horizontal table surface.

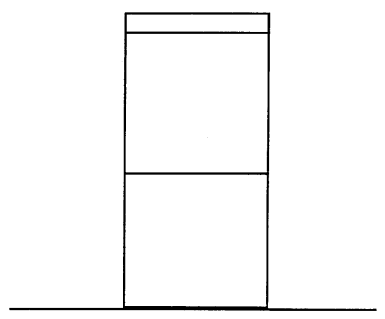

(a)

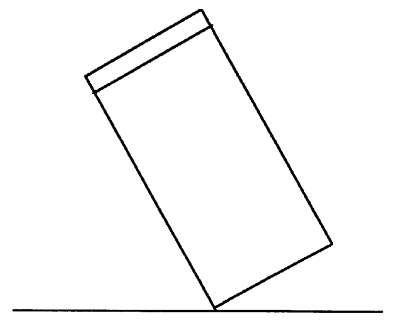

(b)

Figure 1. (a) Drawing of the jar used for the water-level problem. (b) An illustration of a test drawing of a tilted jar. 
2.2.2 Bus task. The standard bus was drawn parked on a 15.5-cm-long flat surface (see figure 2). The bus was a rectangle, $8 \mathrm{~cm}$ by $4.5 \mathrm{~cm}$, with the top two corners beveled (see Robert and Harel 1996). Two circles, $2 \mathrm{~cm}$ in diameter, represented the wheels. A rectangle, $8 \mathrm{~mm}$ by $4 \mathrm{~mm}$, was drawn on the inside roof of the bus to show the 'junction box' where the electrical cord was attached to the roof of the bus. A $7.5-\mathrm{cm}$ long horizontal line segment was used to represent the horizontal at the bottom of the hill. The standard depicted the bus at $0^{\circ}$, and an electrical cord, $2.4 \mathrm{~cm}$ in length, with a circle, $0.6 \mathrm{~cm}$ in diameter, drawn at the end represented the light bulb. The electrical cord was connected to the center of the junction box. The 10-cm-long hills were at four different angles: $15^{\circ}, 30^{\circ}, 45^{\circ}$, and $60^{\circ}$, with $0^{\circ}$ representing the horizontal.

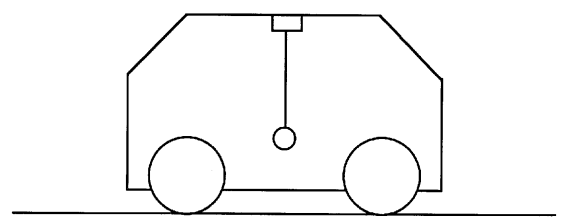

(a)

Figure 2. (a) An illustration of the standard bus. (b) An example of a test stimulus. Drawings of the hills used in the telephone-pole task were like the hills used in the bus task, but without the depiction of the bus.

2.2.3 Telephone-pole task. A 6-cm-long horizontal line segment was connected to the hill, depicted by a 10 -cm-long line. The line representing the hill was drawn at one of four angles $\left(15^{\circ}, 30^{\circ}, 45^{\circ}\right.$, and $60^{\circ}$; with $0^{\circ}$ representing the horizontal).

\subsection{Design and procedure}

The data on the horizontal were treated separately from the judgments of the vertical. The data for judgments of the horizontal were treated as a 2 (gender) $\times 4$ (angle of tilt) design, with repeated measures on angle of tilt. Judgments of the vertical were analyzed as a between - within ANOVA, with the independent groups factors being task (bus vs hills), gender, and repeated measures on angle of tilt.

In all conditions, subjects were asked to make a raised-line drawing to indicate their answers. They were not given feedback, and no time constraints were imposed. All subjects completed the three tasks in the same order, with the jar task first, followed by the bus task, and finally the telephone-pole task. Task order was not varied, since it was thought that this would be too disorienting for the subjects. The drawing task was novel and unfamiliar, and pilot data indicated rather large error scores. Subjects were allowed to redo their drawings until satisfied, but were not allowed to alter previous drawings.

2.3.1 Jar task. Subjects felt the standard drawing and were instructed to guess at the identity of the tangible picture. All drawings were presented flat on the horizontal table surface, with the horizontal lines parallel to the front edge of the table. Many subjects indicated they thought the standard was a picture of furniture, such as a table, or clothing, such as a hat. They were then told the drawing was that of a jar with water in it, sitting on the surface of a table. The blindfolded subjects felt the standard drawing and were instructed to find the parts of the picture representing the cap, the table surface, and the water level, and identify them. This procedure was adopted to be sure that all subjects were able to correctly interpret the drawings during the testing phase of the experiments. Subjects were then told to note that the jar was half-full of water. Next, subjects felt the drawings of the tilted jar, and were asked to identify the cap and the table surface. Before drawing each picture, subjects were reminded that the jar was 
"super-glued in place and not moving". Subjects then drew a tangible line representing the water level in each of the drawings. They were told to draw the water level as it would be in the real world, assuming that the jar was fixed in place for some time. A ruler was visible to subjects before they were blindfolded, and was available for subjects' use if they wished.

2.3.2 Bus task. Subjects first felt the standard drawing, which showed the bus at $0^{\circ}$. An electrical cord, $2.4 \mathrm{~cm}$ in length, with a circle, $0.6 \mathrm{~cm}$ in diameter, drawn at the end represented the light bulb. Subjects were told that the bus was parked and not moving, and had been "sitting there for a few minutes". Subjects were told to find the wheels, the light bulb, the electrical cord, and the junction box. Feedback was given during this familiarization phase, but not during testing. Next, they felt pictures of the bus on the hill, and they were then asked to draw the cord and the light bulb. Subjects were asked to connect the cord to the rectangular junction box hanging from the ceiling of the bus.

2.3.3 Telephone-pole task. Subjects were told to draw a single line to represent a telephone pole on the hill at four different angles. They were told to try to draw the pole as it would be in the real world, in the middle of the hill portion of each drawing. The center of each line representing the hill was at the person's midline, flat on the table surface in front of the subjects.

\section{Results}

The response measure was the absolute angular error. The horizontal task was analyzed separately as a 2 (gender) $\times 4$ (angle) ANOVA. Judgments of the vertical were analyzed separately as a 2 (task) $\times 2$ (gender) $\times 4$ (angle) ANOVA.

\subsection{Jar task}

Subjects generally showed large error scores and were systematically influenced by the angle of the jar (see table 1). A correct response is a line drawn parallel to the table surface. Thus, an error score was calculated by measuring the angle of the subject's line and noting the deviation from the horizontal. A line was drawn to connect the points where the subject's line intersected each side of the jar. The response measure was the absolute value of the angular difference between this line and the horizontal.

Table 1. Haptic jar task and assessment of horizontality: absolute error scores $(M)$, percent correct $(P)$, and mean number correct $(m)$, with standard deviations (SD) for males and females.

\begin{tabular}{lllllll}
\hline & \multicolumn{2}{l}{ Angle of tilt } & $m$ & & \\
\cline { 2 - 5 } & $-60^{\circ}$ & $-30^{\circ}$ & $30^{\circ}$ & $60^{\circ}$ & & \\
\hline Males & & & & & & \\
$M$ & $32.2^{\circ}$ & $19.9^{\circ}$ & $20.5^{\circ}$ & $32.2^{\circ}$ & 0.80 & $20.8 \%$ \\
SD & $23.0^{\circ}$ & $15.9^{\circ}$ & $16.2^{\circ}$ & $24.4^{\circ}$ & 1.1 & \\
Females & & & & & & \\
$M$ & $45.1^{\circ}$ & $28.3^{\circ}$ & $27.8^{\circ}$ & $43.7^{\circ}$ & 0.33 & $8.3 \%$ \\
SD & $22.5^{\circ}$ & $13.8^{\circ}$ & $10.6^{\circ}$ & $17.1^{\circ}$ & 0.9 & \\
\hline
\end{tabular}

A between - within ANOVA showed that the main effect of gender failed to reach significance $\left(F_{1,22}=2.5, p=0.13\right)$. The interaction of gender and angle was also nonsignificant $(F<1)$. Subject responses, however, varied significantly with angle $\left(F_{3,66}=11.6\right.$, $p<0.0001)$. Because the correct answer at each tilt entails an error score of zero, an effect of angle indicates that subjects were influenced systematically by the tilt of the jar. Errors given $-60^{\circ}$ and $-30^{\circ}$ were generally negative degrees deviated from true. A Newman - Keuls a posteriori test showed that jars that were tilted equally (ie $-60^{\circ}$ and $60^{\circ}$ ) gave the same pattern of results (mean absolute error for $-60^{\circ}$ was $38.6^{\circ}$, 
and for $60^{\circ}, 37.9^{\circ}$; for $-30^{\circ}$ it was $24.1^{\circ}$, and for $30^{\circ}$ it was $24.1^{\circ}$ ), regardless of the direction of tilt $(p>0.05)$. All other means were significantly different $(p<0.01)$.

In earlier studies (Rebelsky 1964) the data were analyzed in terms of the number of correct answers (with absolute errors of $5^{\circ}$ or less). The data were reanalyzed with the use of that scoring method. A one-tailed $t$-test (predicting a better score for males) showed a nonsignificant difference between the groups $\left(t_{22}=1.22, p>0.20\right)$.

Many subjects were unable to accurately represent the water surface. Subjects giving incorrect answers generally made one of two types of drawings: lines were either parallel to the top of the jar; or sloping upward, tending toward approaching a line parallel to the side of the jar. Absolute error scores increased as the overall tilt of the jar increased. It should be noted, however, that subjects showed a great deal of variability. On average, males did slightly better than females, but, given the variability of performance, the difference was small and statistically nonsignificant.

\subsection{Bus task}

The response measure was the absolute value of the angular difference between the response and the vertical. In all cases, the correct response was a line perpendicular to the horizontal.

Table 2. Haptic bus task: absolute error scores $(M)$, percent correct $(P)$, mean number correct $(m)$, and standard deviations (SD) in drawings of the vertical by males and females.

\begin{tabular}{|c|c|c|c|c|c|c|}
\hline & \multicolumn{2}{|c|}{ Angle of hill } & \multirow{2}{*}{$\frac{m}{45^{\circ}}$} & \multirow{2}{*}{$\begin{array}{l}P \\
60^{\circ}\end{array}$} & & \\
\hline & $15^{\circ}$ & $30^{\circ}$ & & & & \\
\hline \multicolumn{7}{|c|}{ Males } \\
\hline$M$ & $22.8^{\circ}$ & $31.1^{\circ}$ & $22.8^{\circ}$ & $29.8^{\circ}$ & 1.1 & $27.1 \%$ \\
\hline SD & $15.0^{\circ}$ & $21.1^{\circ}$ & $18.5^{\circ}$ & $27.2^{\circ}$ & 1.5 & \\
\hline \multicolumn{7}{|c|}{ Females } \\
\hline$M$ & $15.3^{\circ}$ & $25.5^{\circ}$ & $24.5^{\circ}$ & $40.6^{\circ}$ & 0.4 & $10.4 \%$ \\
\hline SD & $12.6^{\circ}$ & $14.7^{\circ}$ & $15.3^{\circ}$ & $17.8^{\circ}$ & 0.7 & \\
\hline
\end{tabular}

Table 3. Haptic hill/pole task: absolute error scores $(M)$, percent correct $(P)$, mean number correct $(m)$, and standard deviations (SD) in production of the vertical by males and females.

\begin{tabular}{cccc} 
Angle of hill & $m$ & $P$ \\
\hline $15^{\circ}$ & $30^{\circ}$ & $45^{\circ}$ & $60^{\circ}$
\end{tabular}

\begin{tabular}{lllllll}
\hline Males & & & & & & \\
$M$ & $9.2^{\circ}$ & $15.8^{\circ}$ & $15.0^{\circ}$ & $18.6^{\circ}$ & 1.9 & $47.9 \%$ \\
SD & $9.0^{\circ}$ & $17.2^{\circ}$ & $17.1^{\circ}$ & $20.6^{\circ}$ & 1.5 & \\
Females & & & & & & \\
$M$ & $9.0^{\circ}$ & $9.3^{\circ}$ & $12.6^{\circ}$ & $16.5^{\circ}$ & 1.0 & $25.0 \%$ \\
SD & $6.0^{\circ}$ & $8.6^{\circ}$ & $10.8^{\circ}$ & $12.9^{\circ}$ & 1.0 & \\
\hline
\end{tabular}

A between - within ANOVA revealed highly significant effects of task $\left(F_{1,44}=11.34\right.$, $p<0.01)$, and angle of tilt $\left(F_{3,132}=12.78, p<0.001\right)$, but a nonsignificant effect of gender $(F<1)$. The interaction between task and gender was nonsignificant $(F<1)$, as was the interaction between task and angle $\left(F_{3,132}=2.0, p>0.10\right)$. The interaction between gender and angle was marginal $\left(F_{3,132}=2.55, p=0.058\right)$, as was the interaction between task, gender, and angle $\left(F_{3,132}=2.15, p=0.10\right)$.

Males and females showed very similar performance on judgments of the vertical (mean error for males, $M=20.6^{\circ}$; for females, $M=19.2^{\circ}$ ). Furthermore, errors were much lower for drawing poles on hills $\left(M=13.2^{\circ}\right)$ than in the bus task $\left(M=26.5^{\circ}\right)$. 
These data suggest that subjects may use a body-centered frame of reference which helps them draw the vertical in the hill task (see Millar 1991, 1994).

There are two important differences between the two verticality tasks that could explain the difference in performance levels. First, the bus task provides an oblique framework that subjects must ignore in order to perform the task correctly. In the telephone-pole task, the only distracting oblique line is the actual hill, and subjects can simply use their body midline as a frame of reference to determine the vertical (see Millar 1991, 1994). Subjects are then able to draw a line receding from the body midline. In the bus task, subjects must draw the cord and light bulb inside the boundaries provided by the bus. Subject responses may vary with field dependence or the relative salience of cues, when using haptics. Second, although both tasks involve an understanding of the vertical, several subjects were distracted by the possibility of mobility of the bus. Despite being told that the bus had been "parked on a hill" and that it had been "sitting there for a few minutes", several subjects still asked about the direction in which the bus was moving before it stopped.

It is possible, of course, that practice effects would have led to improved performance on the hill task. However, subjects were not given feedback, and so this explanation of the data seems unlikely, especially given the large improvement from the bus to the hill task.

\section{General discussion}

Gender differences were not found for the haptic drawing version of the water-level task. This result is consistent with the findings of Robert and her colleagues (Berthiaume et al 1993; Robert et al 1994). It should be noted that both males and females had relatively high error scores when judging the vertical with the bus task, but both groups improved considerably when asked to draw a telephone pole. The results suggest that possible gender differences are probably linked to the response measure. Furthermore, it is likely that improved performance with the pole task reflects the greater ease with which subjects could use body-centered spatial reference frames when drawing the vertical (Millar 1991, 1994). As inclines become sharper, it is more difficult to ignore the reference frame provided by a surround. However, this is not the case for drawings of the vertical pole on a hill, and subjects do much better on this task.

Part of the difficulty that some subjects have with the water-level problem may involve difficulty with producing raised-line drawings while blindfolded. Sighted subjects are unfamiliar with the production of raised-line drawings, and may be disoriented initially when blindfolded (Heller 1993). In the rod-and-frame task, Bitterman and Worchel (1958) reported that sighted persons were more disoriented by body tilt than blind subjects when making judgments of the vertical. They concluded that sighted subjects are susceptible to disorientation when denied visual spatial information, reflecting visual dominance in orientation of normal individuals. Also, blindfolded sighted subjects did not do as well as blind subjects when interpreting tangible pictures of a board at a slant, but only for raised-line drawings of the vertical panel (Heller et al 1996b). This argument is also consistent with gender differences that have been obtained in the rod-and-frame task. The behavior and comments of one female subject in the present report would be consistent with this interpretation of the data. She stated that she found the use of blindfolds disorienting. This subject made an error with the first set of choice pictures. On the second set of choice pictures she suddenly recalled the physical principle (horizontality) that the water level is always horizontal, and was correct on all subsequent trials. Performance on these tasks is dependent upon many factors, not only one's knowledge of physics (Robert and Harel 1996). Thus, an individual may understand a spatial concept, yet not show perfect performance.

Consequently, an additional experiment was conducted, with the use of a multiplechoice variant of the water-level problem. Blindfolded subjects (twenty-eight new and 
naive subjects: fourteen male and fourteen female) were initially exposed to the standard drawing, and then to sets of multiple-choice pictures. Each of the four angles $\left(-60^{\circ}\right.$, $-30^{\circ}, 30^{\circ}$, and $60^{\circ}$ ) was presented twice, in a random arrangement. For each angle, four raised-line picture choices were generated, including a correct choice and three additional picture foils. One incorrect choice included a drawing with the water level parallel to the base of the jar. In each of the two other picture choices, the water level was $30^{\circ}$ or $-30^{\circ}$ from the line drawn parallel to the base. Thus, each subject was exposed to eight trials. As in prior research, the subjects were blindfolded throughout, and feedback was not given. Subjects were told that they would feel a set of pictures showing water in a jar, and should pick out the picture that showed "... how the water would be in the real world". As in experiment 1 reported here, subjects were told to imagine the jar was stationary, and that they should think of how the water would eventually wind up in the jar.

Table 4 summarizes the results of the multiple-choice version of the haptic Piagetian water-level problem, and shows little difference in the performance of males (mean number correct $M=4.93$ out of 8 total choices) and females $(M=4.5)$. An ANOVA on mean number correct indicated a nonsignificant effect of gender $(F<1)$. The effect of tilt of the jar was highly significant $\left(F_{3,78}=5.84, p<0.01\right)$, but the interaction between gender and tilt was nonsignificant $\left(F_{3,78}=1.48, p>0.20\right)$.

The multiple-choice version of the water-level problem was replicated with drawings of flasks (with round bottoms) to determine if a reduction in the salience of the raised lines would alter the results. The results for an additional twenty-eight new subjects (fourteen males and fourteen females) were similar to those for the multiple-choice task reported above, since the effect of gender on number correct was nonsignificant $\left(F_{1,26}=2.5, p=0.127\right)$. However, all of the other effects were nonsignificant (all $\left.F \mathrm{~s}<1\right)$.

An ANOVA was performed which compared the data for the two multiple-choice water-level problems (total $N=56$ ). The results were similar to those for each analysis considered separately. The effect of bottle shape (jar versus flask) was nonsignificant $(F<1)$, and the effect of gender failed to reach significance $\left(F_{1,52}=2.57, p>0.10\right)$. However, the effect of angle was highly significant $\left(F_{3,156}=4.41, p<0.01\right)$, reflecting lower performance for greater inclines. The interaction between shape and angle was also significant $\left(F_{3,156}=2.79, p<0.05\right)$, with tests of simple effects showing significantly lower performance for the sharper inclines and the jar $\left(F_{3,156}=6.57, p<0.001\right)$, but not for the flask $(F<1)$. The shape by gender interaction was nonsignificant $(F<1)$, as was the interaction between gender and angle $\left(F_{3,156}=1.91, p=0.13\right)$. The interaction between gender, shape, and angle failed to reach significance $(F<1)$.

The results of the multiple-choice tasks are consistent with the idea that gender differences in spatial tasks, like those devised by Piaget, are inconsistent and influenced

Table 4. Piagetian multiple-choice task: mean number correct $(M)$, standard deviation (SD), and percent correct $(P)(N=28 ; 14$ males, 14 females $)$.

\begin{tabular}{lcccc}
\hline & \multicolumn{4}{c}{ Angle of water line in the jar } \\
\cline { 2 - 4 } & $-60^{\circ}$ & $-30^{\circ}$ & $30^{\circ}$ & $60^{\circ}$ \\
\hline Males & & & & \\
$M$ & 1.57 & 0.79 & 1.00 & 1.57 \\
SD & 0.65 & 0.80 & 0.96 & 0.65 \\
$P$ & $79 \%$ & $40 \%$ & $50 \%$ & $79 \%$ \\
Females & 1.07 & 0.93 & 1.07 & 1.43 \\
$M$ & 0.83 & 0.62 & 0.62 & 0.76 \\
SD & $54 \%$ & $47 \%$ & $54 \%$ & $72 \%$ \\
$P$ & Note: Total mean & number correct possible & $M=8$. \\
\hline
\end{tabular}


by a number of variables. Any obtained gender differences were small in magnitude, and could be affected by the response measure that was used. The results from the multiplechoice task were consistent with a lack of gender differences that were obtained in a drawing task, and the negative report by Robert and her colleagues. The only gender differences that appeared in the present study involved a marginally significant interaction between gender and angle in judgments of the vertical. Perhaps some females are slightly more subject to being thrown off by context effects.

One reviewer suggested that one should exercise considerable caution when trying to interpret studies of gender, because gender cannot be manipulated experimentally. It is an individual difference variable, which might require relatively large samples from representative populations. The present data are certainly consistent with this analysis, especially given the largely nonsignificant effects of gender over different tasks.

It should be noted that the present results are not consistent with the idea that information from tangible raised-line drawings is not perceptually salient for touch. This argument could not explain the large differences that were found between judging the vertical in the bus task and for the telephone pole on a hill. Thus, subjects are influenced by the spatial reference information provided by the drawings. Subjects are sometimes better able to ignore distracting cues in haptic conditions, as when drawing telephone poles on a hill.

Acknowledgements. Preparation of this report and some of the research were supported by NIH Grant 2 SO6 RR-08040 and NIH RO1 EY12040-01. We are grateful to Cindy Flynt for assistance with data collection and analyses.

\section{References}

Berthiaume F, Robert M, St-Onge R, Pelletier J, 1993 "Absence of a gender difference in a haptic version of the water-level task" Bulletin of the Psychonomic Society 31 57-60

Bitterman M E, Worchel P, 1958 "The phenomenal vertical and horizontal in blind and sighted subjects" American Journal of Psychology $66598-602$

Harris L J, Hanley C, Best C T, 1977 "Conservation of horizontality: Sex differences in sixth graders and college students", in Readings in Child Development and Relationships Eds R C Smart, M S Smart (London: Macmillan) pp 375-387

Heller M A, 1993 "Influence of visual guidance on braille recognition: Low lighting also helps touch" Perception \& Psychophysics $54675-681$

Heller M A, Calcaterra J A, Burson L L, Tyler L A, 1996a "Tactual picture identification by blind and sighted people: Effects of providing categorical information" Perception \& Psychophysics $58310-323$

Heller M A, Calcaterra J A, Tyler L A, Burson L L, 1996b "Production and interpretation of perspective drawings by blind and sighted people" Perception 25321 - 334

Heller M A, Schiff W (Eds), 1991 The Psychology of Touch (Hillsdale, NJ: Lawrence Erlbaum Associates)

Millar S, 1991 "A reverse lag in the recognition and production of tactual drawings: Theoretical implications for haptic coding", in The Psychology of Touch Eds M A Heller, W Schiff (Hillsdale, NJ: Lawrence Erlbaum Associates) pp 301-325

Millar S, 1994 Understanding and Representing Space: Theory and Evidence from Studies with Blind and Sighted Children (Oxford: Oxford University Press)

Piaget J, Inhelder B, 1956 The Child's Conception of Space (New York: W W Norton)

Rebelsky F, 1964 "Adults perception of the horizontal" Perceptual and Motor Skills $19371-374$

Robert M, Harel F, 1996 "The gender difference in orienting liquid surfaces and plumb-lines: Its robustness, its correlates, and the associated knowledge of simple physics" Canadian Journal of Experimental Psychology $50280-314$

Robert M, Ohlman T, 1994 "Water-level representation by men and women as a function of rod-andframe proficiency and visual and postural information" Perception 231321 - 1333

Robert M, Pelletier J, St-Onge R, Berthiaume F, 1994 "Women's deficiency in water-level representation: Present in visual conditions yet absent in haptic contexts" Acta Psychologica 87 19-32

Voyer D, Voyer S, Bryden M P, 1995 "Magnitude of sex differences in spatial abilities: A metaanalysis and consideration of critical variables" Psychological Bulletin $117250-270$ 